\title{
Imaging laser Doppler velocimetry
}

\section{Journal Article}

\section{Author(s):}

Meier, Alexander H.; Rösgen, Thomas

Publication date:

2012-04

\section{Permanent link:}

https://doi.org/10.3929/ethz-b-000041612

Rights / license:

In Copyright - Non-Commercial Use Permitted

Originally published in:

Experiments in Fluids 52(4), https://doi.org/10.1007/s00348-011-1192-1 


\title{
Imaging laser Doppler velocimetry
}

\author{
Alexander H. Meier - Thomas Roesgen
}

Received: 17 October 2010/Revised: 9 August 2011/Accepted: 18 August 2011/Published online: 31 August 2011 (C) Springer-Verlag 2011

\begin{abstract}
Imaging laser Doppler velocimetry (ILDV) is a novel flow measurement technique, which enables the measurement of the velocity in an imaging plane. It is an evolution of heterodyne Doppler global velocimetry (HDGV) and may be regarded as the planar extension of the classical dual-beam laser Doppler velocimetry (LDV) by crossing light sheets in the flow instead of focused laser beams. Seeding particles within the flow are illuminated from two different directions, and the light scattered from the moving particles exhibits a frequency shift due to the Doppler effect. The frequency shift depends on the direction of the illumination and the velocity of the particle. The superposition of the two different frequency-shifted signals on the detector creates interference and leads to an amplitude modulated signal wherein the modulation frequency depends on the velocity of the particle. This signal is detected using either a high-speed camera or alternatively a smart pixel imaging array. This detector array performs a quadrature detection on each pixel with a maximum demodulation frequency of $250 \mathrm{kHz}$. To demonstrate the feasibility of the technique, two experiments are presented: The first experiment compares the measured velocity distribution of a free jet using ILDV performed with the smart pixel detector array and a high-speed camera with a reference measurement using PIV. The second experiment shows an advanced setup using two smart pixel detector arrays to measure the velocity distribution on a
\end{abstract}

\footnotetext{
A. H. Meier $(\bowtie) \cdot$ T. Roesgen

Institute of Fluid Dynamics, ETH Zurich, Sonneggstrasse 3, 8092 Zurich, Switzerland

e-mail: meier@ifd.mavt.ethz.ch

T. Roesgen

e-mail: roesgen@ifd.mavt.ethz.ch
}

rotating disk, demonstrating the potential of the technique for high-velocity flow measurements.

\section{Introduction}

The measurement of flow velocity distributions based on optical Doppler shift detection remains an active field of research in flow diagnostics. By measuring the frequency shift of light scattered from moving particles, Doppler global velocimetry (DGV) avoids some of the restrictions of particle image velocimetry (PIV). Without the need for resolving individual particles, the method is particularly well suited for large-scale applications. Standard DGV techniques rely on the use of molecular line filters to convert the frequency shift into a change in intensity (Ainsworth et al. 1997; Elliott et al. 1999; Samimy et al. 2000). The technique has been successfully applied in fullscale wind tunnels (Beutner et al. 1998), high-speed flows (Smith et al. 1996) and combustion measurements (Roehle et al. 2000). Molecular line filters can also be used to change the Doppler frequency shift into a phase shift as it is done in near resonant interferometry (Landolt et al. 2009) to measure velocity distributions. However, those methods usually suffer from uncertainties in the order of a few $\mathrm{m} / \mathrm{s}$ (Elliott et al. 1999). With heterodyne Doppler global velocimetry (HDGV), we proposed a new approach to measure global Doppler frequency shifts (Meier et al. 2009). In HDGV, the flow is illuminated using a light sheet as in conventional DGV. The frequency shift of the light scattered from particles within the flow is demodulated using a reference beam from the same coherent light source and detected using a smart pixel detector array (SPDA). This setup can be considered as the planar imaging extension of the original reference beam LDV (Yeh et al. 1964). The 
feasibility of HDGV was successfully demonstrated, but the measured velocities were limited to a maximum value of $2 \mathrm{~mm} / \mathrm{s}$ due to the high modulation frequencies in the signal generated by this optical arrangement. To extend this limited velocity range, imaging laser Doppler velocimetry (ILDV) uses a setup that is the corresponding extension of dual-beam LDV (vom Stein et al. 1969) to planar measurement geometries. Instead of crossing two focused laser beams from the same source, two light sheets are crossed allowing the measurement of Doppler shifts in a planar volume. With two different crossed light sheet configurations, either the out-of-plane velocity or one component of the in-plane velocity become measurable.

\section{Method}

\subsection{Signal formation}

The frequency $v$ of the light scattered from an illuminated moving particle is shifted proportional to its velocity, according to Eq. 1.

$v-v_{0}=v_{d}=\frac{v_{0}}{c}(\mathbf{O}-\mathbf{I}) \cdot \mathbf{V}$.

The Doppler frequency shift $v_{d}$ depends on the magnitude and direction of the velocity $\mathbf{V}$ of the scattering particle, the frequency $v_{0}$ and the direction of the illumination $\mathbf{I}$, the direction of observation $\mathbf{O}$ and the speed of light c. Figure 1 shows the geometry of this scattering process indicating the orientation of the vectors of Eq. 1. In HDGV, the interference between this frequency-shifted light and a reference beam from the same source on the detector is used to demodulate the frequency shift. The result of this superposition is a signal that is modulated with $v_{d}$. The maximum measurable Doppler shift is limited by the detector, while the maximum measurable velocity can be increased by adjusting the geometrical arrangement of $\mathbf{O}$ and $\mathbf{I}$. This arrangement of $\mathbf{O}$ and $\mathbf{I}$ is, however, limited by the imaging system: To measure high velocities, the observation angle has to be very shallow to reduce the sensitivity of the system. To achieve a sensitivity of $50 \mathrm{kHz} /(\mathrm{m} / \mathrm{s})$, which would extend the measurement range of HDGV to approx. $7 \mathrm{~m} / \mathrm{s}$, the angle between $\mathbf{O}$ and I needs to be approx. $2^{\circ}$. This makes it difficult to maintain the whole illuminated plane in focus even with a Scheimpflug imaging arrangement.

To overcome this limitation, a different illumination and demodulation approach is used in dual-beam LDV and ILDV. The particle is illuminated from two different directions $\mathbf{I}_{\mathbf{1}}$ and $\mathbf{I}_{\mathbf{2}}$ simultaneously. The light scattered in the direction of observation $\mathbf{O}$ contains two different frequency shifts $v_{1}$ and $v_{2}$. The square-law detection of

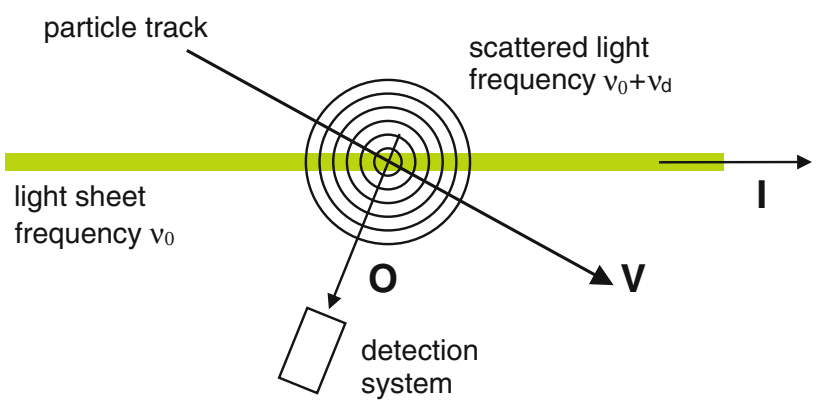

Fig. 1 Geometry of the scattering process

those signals on the detector results in the observable signal $S$,

$S=A_{1}^{2}+A_{2}^{2}+2 A_{1} A_{2} \cos \left(2 \pi\left(v_{1}-v_{2}\right) t+\phi\right)$

$A_{1}$ and $A_{2}$ denote the intensity of the scattered light of the two illumination directions and $\phi$ a random phase shift. The signal $S$ has a constant offset $\left(A_{1}^{2}+A_{2}^{2}\right)$ and is modulated by the difference of the two Doppler shifts. Using Eq. 1, this difference can be written as:

$\Delta v=v_{1}-v_{2}=\frac{v_{0}}{c}\left(\mathbf{I}_{1}-\mathbf{I}_{2}\right) \cdot \mathbf{V}$

The modulation frequency of the signal $S$ no longer depends on the observation direction but only on the crossing angle $\theta$ between the two illumination directions and the velocity of the particle. In dual-beam LDV, the illumination is achieved by crossing two focussed laser beams. For ILDV, this has to be extended to generate a planar illumination by crossing light sheets. Crossing two light sheets to form a measurement plane can be done in different ways. The two most advantageous configurations are shown in Fig. 2. A configuration with two co-planar light sheets is shown in Fig. 2a. Both light sheets illuminate the same plane, but each point in the plane is illuminated from two directions. With this configuration, one of (a)

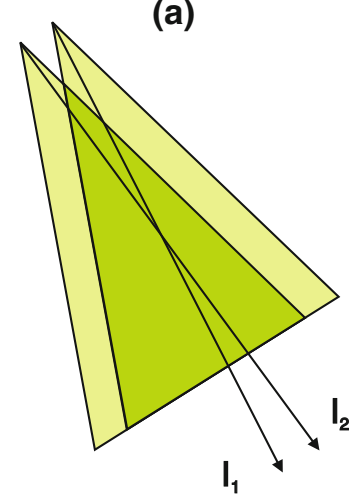

(b)

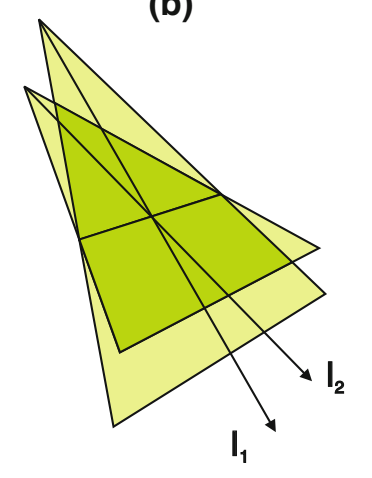

Fig. 2 Different light sheet geometries. (a) co-planar light sheets, (b) crossed light sheets 


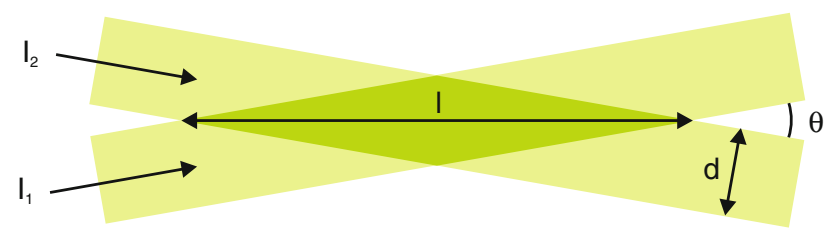

Fig. 3 Close-up on the cross-section of a crossed light sheets setup

the in-plane components of the velocity can be measured. Figure $2 \mathrm{~b}$ shows a configuration with two crossed light sheets that are not in the same plane. Usually, the crosssection of two planes is a line. The intersection of the two light sheets expands from a single line into a volume, however, when very shallow crossing angles and light sheets with a thickness of several millimeters are used. With this configuration, the out-of-plane component of the velocity can be measured. A close-up on the cross-section between the two planes is shown in Fig. 3. While the width of the measurement volume is given by the width of the light sheets, its length $l$ depends on $\theta$ and on the light sheet thickness $d$. For a crossing angle of, e.g., $1^{\circ}$ and a light sheet thickness of $5 \mathrm{~mm}$, the length of the measurement volume is approx. $0.25 \mathrm{~m}$.

By adjusting the angle $\theta$ between the two illumination directions, the sensitivity of the system is adjusted. However, there is a lower limit for the crossing angle: The duration of the signal generated by a particle for one pixel is limited by the transit time $T$ of the particle through the volume that is imaged onto this pixel. This transit time limits the accuracy of the frequency estimation since the finite transit time leads to a spectral broadening $\Delta f$ of the signal:

$\Delta f \sim \frac{1}{T}$

To limit this spectral broadening to $<5 \%$ compared to the signal frequency generated by the particle, it has to generate a signal with at least 20 cycles while it stays on one pixel. While $T$ only depends on the velocity of the particle, the number of cycles the particle generates also depends on the crossing angle: The lower the angle the lower the frequency of the signal is for a given velocity, and thus, the lower the number of cycles generated during $T$. But this limitation also shows an interesting property of the measurement technique: The larger the measurement area is the lower the crossing angle can be since the transit time $T$ is increased as well.

\subsection{Detection}

Using crossing angles of approx. $1^{\circ}$, the modulation frequencies of the signal are in the order of $30 \mathrm{kHz} /(\mathrm{m} / \mathrm{s})$. Those frequencies can be detected using the latest high-speed cameras (HSC), which offer frame rates up to 1 million frames per second (Mfps). But in exchange to the high frame rate, their resolution drops down from usually around $1,000 \times 1,000$ pixels to small subwindows or narrow stripes since their speed is limited by the readout speed of the sensor.

Another approach for the detection is the use of a socalled smart pixel detector array (SPDA), which has been developed for parallel optical coherence tomography (Beer et al. 2005). The advantage of this detector array is that it not only detects the light as does a conventional integrating camera but also performs a dual-phase lock-in detection on each pixel with a maximum demodulation frequency $v_{D}$ of $250 \mathrm{kHz}$ at a resolution of $144 \times 90$ pixels. Using the signal processing described in Sect. 2.3, this compares to a frame rate of $1 \mathrm{Mfps}$ for a conventional high-speed camera. As it is developed to detect signals with a low modulation depth, it additionally offers an offset compensation that increases the sensitivity to $86 \mathrm{~dB}$, which is helpful if many particles are imaged onto the same pixel, and thus, the modulation depth of the signal is low.

For the experiments presented in Sect. 3, the SPDA detector array was used due to its potential to detect signals with modulation frequencies up to $500 \mathrm{kHz}$ with a reasonable resolution. Nevertheless, use of a conventional high-speed camera for ILDV is also possible as will be shown in Sect. 3.1 as well.

\subsection{Signal processing}

The signal processing of the data obtained from the SPDA strongly depends on the on-pixel preprocessing of the detected signal. Thus, the operating principle of the sensor is explained in detail first followed by the subsequent postprocessing of the data obtained by the SPDA.

The SPDA performs a dual-phase lock-in detection on each pixel. Dual-phase lock-in amplifiers are normally used to extract the amplitude $A$ and the phase $\phi$ of weak periodic signals of known frequency from a noisy background. The functional principle of a dual-phase lock-in amplifier relies on the orthogonality of sinusoidal functions as described in Eqs. 5 and 6:

$$
\begin{aligned}
I & =\left\langle A \cos (2 \pi \Delta v t+\phi) \cos \left(2 \pi v_{D} t\right)\right\rangle \\
& =\left\{\begin{array}{cc}
\frac{A}{2} \cos (\phi) & \text { if } v_{D}=\Delta v \\
0 & \text { otherwise }
\end{array}\right. \\
Q & =\left\langle A \cos (2 \pi \Delta v t+\phi) \sin \left(2 \pi v_{D} t\right)\right\rangle \\
& =\left\{\begin{array}{cc}
\frac{A}{2} \sin (\phi) & \text { if } v_{D}=\Delta v \\
0 & \text { otherwise }
\end{array}\right.
\end{aligned}
$$

The periodic input signal with the frequency $\Delta v$, phase $\phi$ and amplitude $A$ is multiplied with a periodic reference signal at the demodulation frequency $v_{D}$ and averaged over 
a sufficiently long time. The output of the lock-in amplifier is only non-zero if the signal frequency $\Delta v$ and the demodulation frequency $v_{D}$ are the same. The resulting inphase $(I)$ and quadrature $(Q)$ components can be used to calculate the amplitude $A$ and the phase $\phi$ of the signal:

$A=\sqrt{I^{2}+Q^{2}}$

$\phi=\arctan \left(\frac{Q}{I}\right)$

This lock-in detection is implemented for each pixel on the SPDA as follows: The incoming light is first integrated on the photosensitive area of the pixel and sampled at four times the demodulation frequency $v_{D}$. These samples are then multiplied by a discrete periodic signal with frequency $v_{D}$ and the discrete values $[1,0,-1,0]$ for the $I$ component and for the $Q$ component with the values $[0,1,0,-1]$. These discrete values replace the sine and the cosine of the lock-in detection algorithm. The signals are then averaged over several periods and read out. Equations 9 and 10 describe this process:

$I=\sum_{i=0}^{N-1}\left(\int_{4 i T_{q p}}^{(4 i+1) T_{q p}} S(t) \mathrm{d} x-\int_{(4 i+2) T_{q p}}^{(4 i+3) T_{q p}} S(t) \mathrm{d} x\right)$

$Q=\sum_{i=0}^{N-1}\left(\int_{(4 i+1) T_{q p}}^{(4 i+2) T_{q p}} S(t) \mathrm{d} x-\int_{(4 i+3) T_{q p}}^{(4 i+4) T_{q p}} S(t) \mathrm{d} x\right)$

$S(t)$ is the signal to be detected, $T_{q p}$ denotes the integration time $\left[T_{q p}=1 /\left(4 v_{D}\right)\right]$ and $N$ is the number of periods to be averaged. The averaging process cannot be performed over infinitely long times. The effect of the finite averaging for three different averaging lengths $N$ on the calculated amplitude for an input signal $S$ with $A_{1}=A_{2}=1$ and frequency $\Delta v$ is shown in Fig. 4. The sharp discrimination described in Eqs. 5 and 6 between signals that are modulated with $v_{D}$ and signals that are modulated with a different frequency is relaxed: Signals with frequencies different from the demodulation frequency $v_{D}$ also generate a non-zero output signal. To achieve a peak-width narrower than $1 \%$, the averaging has to be performed over more than $N=40$ periods.

Thus, using the detector as a lock-in amplifier for a flow measurement does not offer a practical performance: For a measurement resolution of $1 \%$ of the measured frequency range, a sweep of the detector is needed with 100 different lock-in frequencies and an averaging over 40 periods for each frequency. This leads to long acquisition times to obtain a reasonably accurate result.

Another more promising use of the demodulation principle is based on the pulse pair statistics method described by Miller et al. (1972): The Wiener-Khinchin theorem

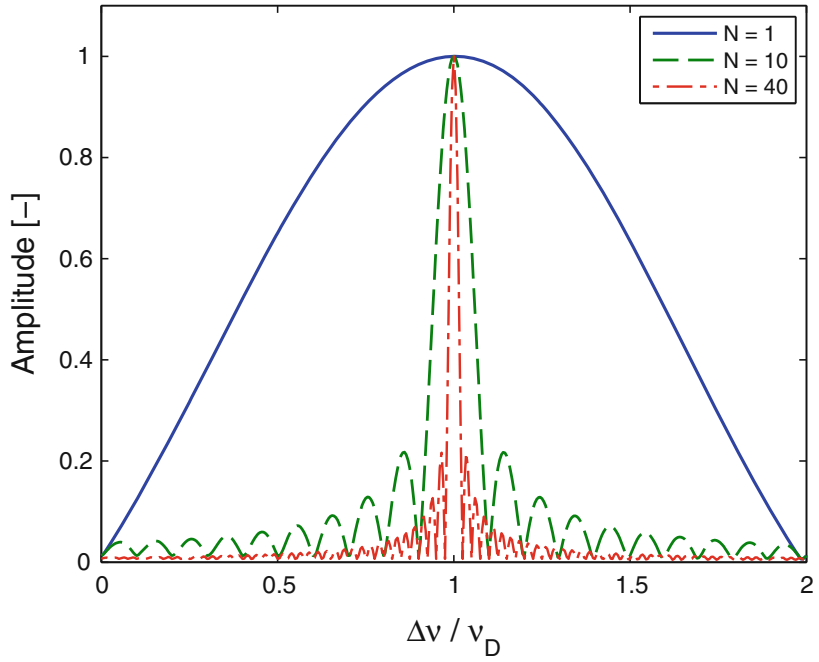

Fig. 4 Output amplitude of the lock-in detection as a function of the signal frequency for different averaging lengths

states that the power spectral density $W(f)$ and the corresponding autocorrelation $R(\tau)$ of a signal are Fourier transform pairs, and the mean $\mu$ of $W(f)$ corresponds to the derivative of the argument $\phi(\tau)$ of the complex autocorrelation $R(\tau)$ evaluated at $\tau=0$ :

$\mu(W(f))=\frac{\int_{-\infty}^{\infty} W(f) f \mathrm{~d} f}{\int_{-\infty}^{\infty} W(f) \mathrm{d} f}=\left.\frac{1}{i 2 \pi R(0)} \frac{\mathrm{d} R(\tau)}{\mathrm{d} \tau}\right|_{\tau=0}$.

Since the autocorrelation is a Hermitian function, this can be reduced to

$\mu(W(f))=\dot{\phi}(0)$.

For small $\tau \neq 0, \mu(W(f))$ can be estimated by

$\mu(W(f))=\dot{\phi}(0) \approx \frac{\phi(\tau)}{\tau}=-\frac{1}{2 \pi \tau} \arctan \left(\frac{\Im\{R(\tau)\}}{\Re\{R(\tau)\}}\right)$

This estimate of the mean frequency is only useful for zero mean analytic signals since the power spectral density of real signals is symmetric, i.e., $W(-f)=W(f)$ and thus $\mu(W(f))=0$. Combining the two output signals $I$ and $Q$ of the detector into a complex signal $C$

$C(t)=I(t)+i Q(t)$

with the corresponding autocorrelation $R(\tau)$

$R(\tau)=\langle C(t) C(t+\tau)\rangle$

results in a zero mean signal with an asymmetric power spectral density. Thus, the concept of evaluating the first derivative of the autocorrelation to estimate the mean frequency in the signal is valid. However, the estimate based on Eq. 13 is only accurate for modulation frequencies $\Delta v$ close to the demodulation frequency $v_{D}$. To expand the 
applicable frequency range of the algorithm, a calibration function for the mean frequency $\mu$ is needed. This calibration function can be derived by inserting Eq. 2 into Eqs. 9, 10, 14, 15 and 13 and leads to Eq. 16.

$\mu=\frac{-1}{2 \pi \tau} \arctan \left(\tan (2 \pi \Delta v \tau) \sin \left(2 \pi \Delta v T_{q p}\right)\right)$

The calculated mean frequency $\mu$ only depends on the integration time $T_{q p}$, and the time delay $\tau$ used to evaluate the autocorrelation $R(\tau)$. The influence of different time delays $\tau$ on $\mu$ is shown in Fig. 5. For $\tau=T_{q p}$, it is a strictly increasing function. Numerical inversion leads to the required relationship between the calculated mean frequency $\mu$ and the present signal frequency $\Delta v$. For $\tau=4 T_{q p}$ and $\tau=8 T_{q p}$, the mean frequency shows an additional wrapping. For flow measurements, this wrapping is less problematic than it seems: A flow field usually does not show such strong discontinuities, and therefore, the flow field can be reconstructed. The phase unwrapping problem can be solved in a least-squares sense using for example the fast cosine transforms as suggested by Ghiglia et al. (1994).

This data analysis scheme is attractive as it can detect a complete range of frequencies without a systematic and time-consuming frequency sweep, but it requires a set of image pairs acquired with a small time delay $\tau$. Those image pairs can be two consecutive images of one SPDA. The time delay $\tau$ is then limited by the frame rate of the SPDA of max. 6,000 fps. Alternatively, the image pairs can be acquired using two SPDAs where one detector array operates with a fixed delay with respect to the other. A system using only one SPDA is presented in Sect. 3.1, and the benefits of a dual SPDA camera system will be shown in Sect. 3.2.

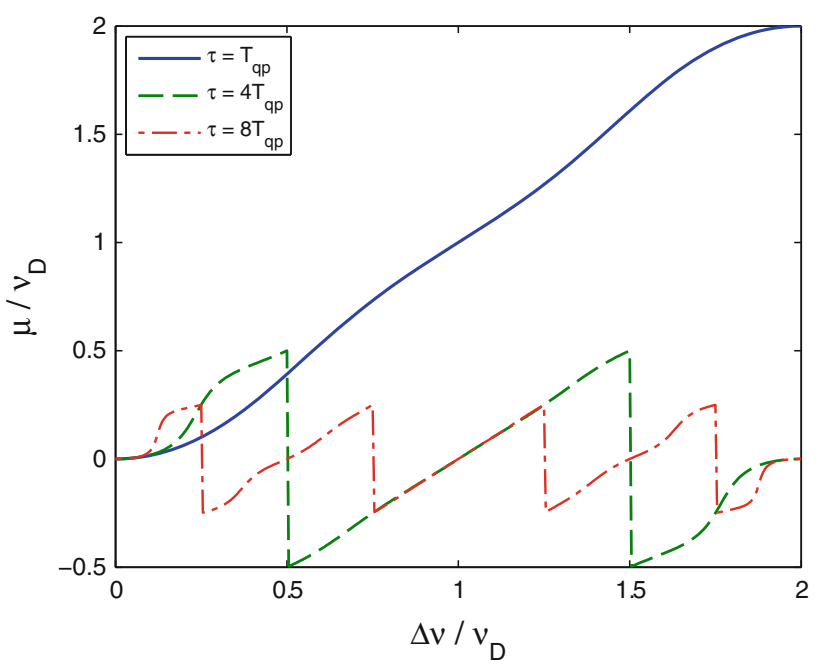

Fig. 5 Estimated mean frequency $\mu$ as a function of the real frequency $\Delta v$ for different time delays $\tau$
In contrast, analyzing data recorded with a high-speed camera is more straightforward. Since such a camera records just a stream of images, the signal of each pixel can be analyzed using a wide range of frequency detection algorithms as it is done in single-point LDV systems. For the results presented in Sect. 3.1, two different algorithms are used. To assess the applicability of the signal processing presented for the SPDA, the data of the HSC are analyzed using the same algorithm. This result is compared with the standard approach of a fast Fourier transform followed by a least-squares peak interpolation with a Gaussian function.

Once the frequency of the Doppler shift is extracted from the data, it has to be converted to a velocity using Eq. 3. Due to the planar expansion of the measurement plane, the angle between the two illumination directions $\mathbf{I}_{\mathbf{1}}$ and $\mathbf{I}_{\mathbf{2}}$ varies over the measurement plane. This variation can be calibrated by manually measuring the crossing angle in several locations. Due to the small crossing angles, this is a very tedious and error-prone procedure. A faster and more accurate approach is an optical calibration of the system using a checkerboard target, which is placed in the field of view of the camera system and recorded before or after the measurement. The angle between the two illumination directions is then measured only at one reference location on the checkerboard, and the image of the checkerboard is used to calculate the change of the crossing angle relative to the reference location for each pixel.

\section{Measurements}

\subsection{Free jet experiment}

To demonstrate the feasibility of a flow measurement using ILDV with one SPDA or a high-speed camera, the velocity distribution of a free jet is measured simultaneously with ILDV and PIV. The setup used for this experiment is shown in Fig. 6. The experiment is performed inside an acrylic glass box (side length $1 \mathrm{~m}$ ). This allows for a homogeneous and continuous seeding of the jet and the quiescent air around it, and the acrylic glass walls allow for an easy optical access for the illumination and the cameras. The jet is generated using a fan at the inlet of the black tube located at the bottom of the acrylic glass box. The flow is then redirected by $90^{\circ}$ to obtain a vertical jet. This redirection is necessary to have a long enough inflow in order to reduce the influence of the fan on the flow profile and to keep the jet nozzle close enough at the bottom of the acrylic glass box to have a large enough measurement area above it. The vortices generated in the flow by the $90^{\circ}$ bend are suppressed by a honeycomb grid right after the bend. Nevertheless, it influences the 


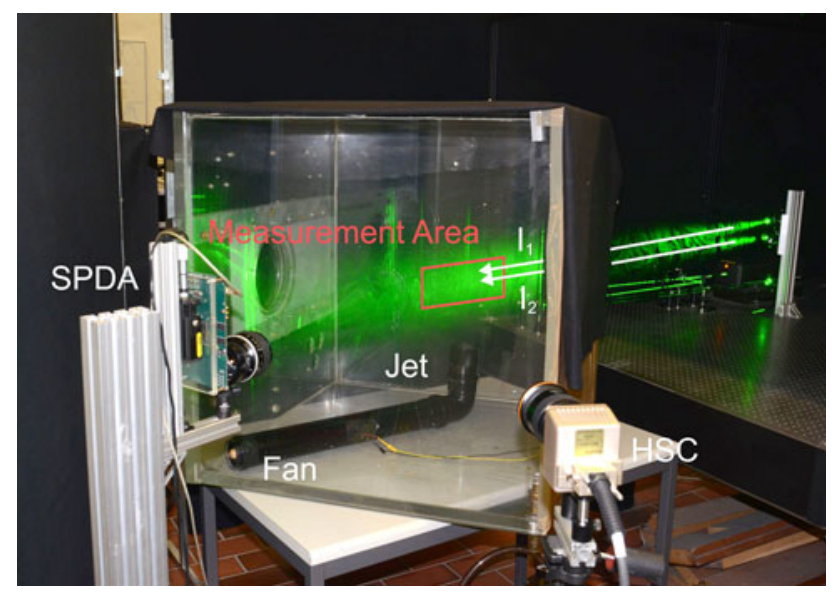

Fig. 6 Setup of the simultaneous ILDV and PIV measurement

resulting jet profile as will be seen in the results. The two cameras used for the simultaneous ILDV and PIV measurements are located outside of the acrylic glass box. The SPDA on the left is equipped with a Nikon $85 \mathrm{~mm} f / 1.4$ lens. Due to its low fill factor of only $10 \%$, a very shallow observation angle of $25^{\circ}$ is necessary to receive enough light from the micron-sized scattering particles. The Mie scattering efficiency in this direction is much larger compared to a more orthogonal viewing arrangement. To keep the whole light sheet in focus, the sensor and the lens are set up in a Scheimpflug imaging arrangement. The highspeed camera (HSC, Photron Fastcam Ultima 512, Tokina 28-70 mm f/2.8) for the PIV measurements can be placed with a less extreme observation angle of approx. $80^{\circ}$. Nevertheless, a Scheimpflug optical arrangement is necessary as well. The flow is illuminated from the right using two cylindrical lenses. A close-up of the optical setup is shown in Fig. 7. The laser beam of the $\mathrm{CW}$ laser (Coherent Verdi V5, $\lambda_{0}=532 \mathrm{~nm}, 5.5 \mathrm{~W}$ ) is split up into two separate beams using a 50:50 beam splitter (BS) and then redirected using several mirrors to two cylindrical lenses (CL, $f=-50 \mathrm{~mm}$ ), which expand the two laser beams to light sheets with a thickness of approx. $5 \mathrm{~mm}$ and cross them inside the acrylic glass box. With the cylindrical lenses placed above each other generating vertical light sheets, this arrangement corresponds to the co-planar light sheet geometry in Fig. 2a. Thus, one of the in-plane components namely the z-component of the velocity can be measured. The measurement area is about $180 \mathrm{~mm} \times 40 \mathrm{~mm}$ large and is located about $200 \mathrm{~mm}$ above the orifice of the jet. The crossing angle between the two illumination directions varied over the field of view from $2.1^{\circ}$ to $2.3^{\circ}$.

The volume inside the acrylic glass box is seeded using a Laskin nozzle generating particles with a diameter of approx. $1 \mu \mathrm{m}$. The seeding density has to be kept as low as

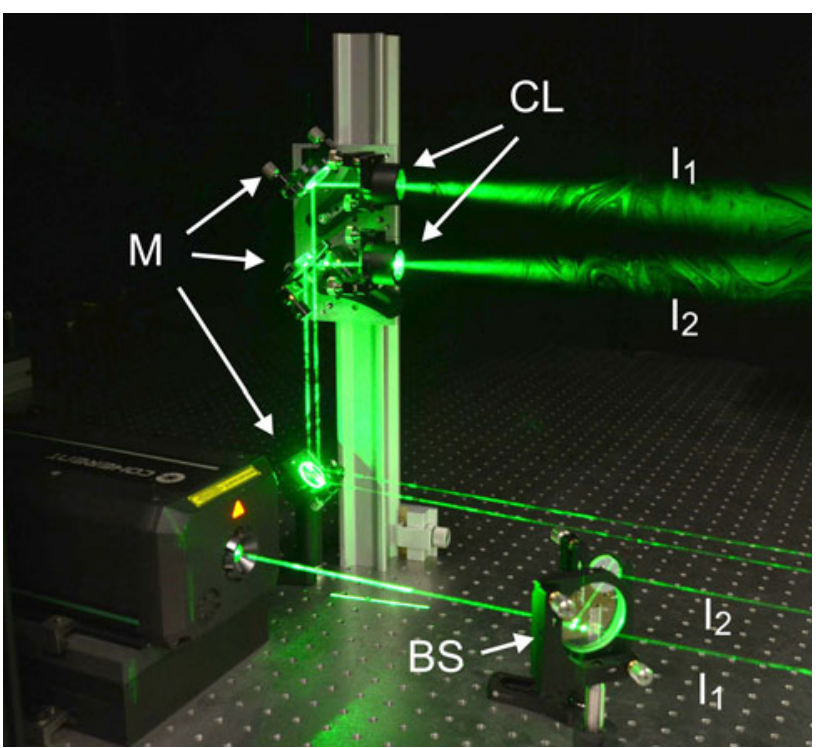

Fig. 7 Close-up on the illumination of the simultaneous ILDV and PIV measurement

possible: There should be just enough particles in the measurement volume to generate a detectable continuous signal. Increasing the seeding density above this level does not improve the signal quality. The superposition of the signal of several particles leads to an increased offset, while the modulation depth of the signal decreases. This is due to the random phase $\phi$ of the signal scattered from each particle described by Eq. 2 .

For the measurement, the camera recorded 512 images with a frame rate of $500 \mathrm{fps}$ and the SPDA was set to a demodulation frequency $v_{D}$ of $4 \mathrm{kHz}$ and recorded 299 images with a frame rate of $2000 \mathrm{fps}$ resulting in a time delay $\tau=8 T_{q p}=500 \mu \mathrm{s}$. After the measurement, the checkerboard target for the optical calibration of both measurement systems was placed inside the acrylic glass box and recorded with both cameras. With the checkerboard target inside the box, the crossing angle between the two illumination directions is measured at the edge of the target by measuring the distance between the two light sheets right at the two cylindrical lenses and the distance to the edge of the target.

The data obtained by the SPDA are then processed as described in Sect. 2.3: First, the signal frequency on each pixel is determined and then using the checkerboard target the angle between the illumination directions is calculated, and the measurement data are mapped onto a regular coordinate system.

The PIV analysis of the data from the HSC is performed using the commercial software PIVView (PIVTec, Germany). The pixel shifts obtained from the PIV analysis are converted into velocities and mapped onto the same regular 


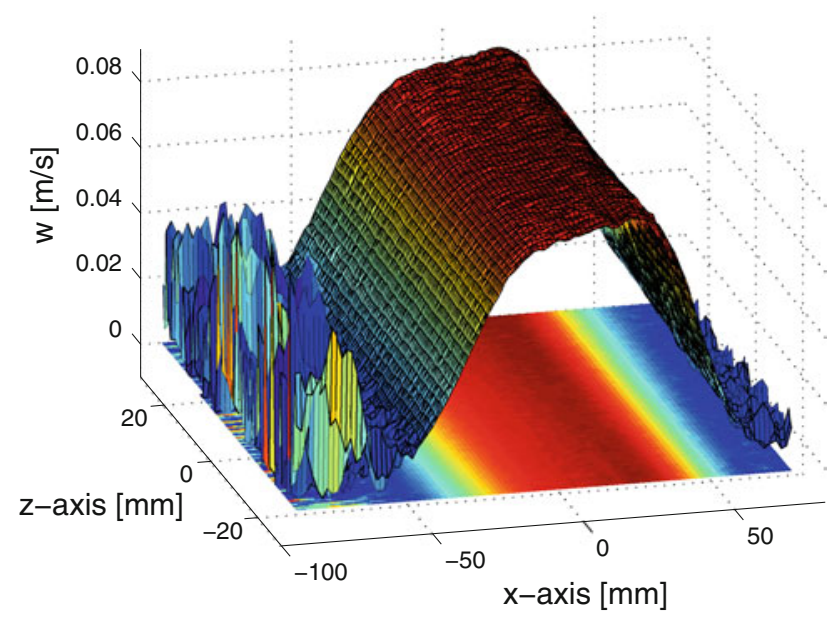

Fig. 8 Measured velocity distribution of the jet using ILDV

coordinate system as the ILDV data using the checkerboard target reference data.

The result of the ILDV measurement is shown in Fig. 8. The shape of the jet is clearly visible, and it is slightly asymmetrical due to the $90^{\circ}$ bend at the bottom of the box. Due to its low velocity, the jet is more or less laminar and its shape changes only slightly along the z-axis. On both sides of the jet, where the velocity drops to zero, the signal becomes noisy. This is due to the fact that zero velocity creates zero Doppler shift, and the camera noise becomes the dominating part of the signal.

The comparison between the ILDV measurement and the PIV measurement is shown in Fig. 9. It shows the velocity distribution in a cross-section of the jet. For velocities faster than $1 \mathrm{~cm} / \mathrm{s}$, the ILDV measurement shows a very good agreement with the PIV measurement. For velocities slower than $1 \mathrm{~cm} / \mathrm{s}$, the disturbance of the measurement by the camera noise is again visible.

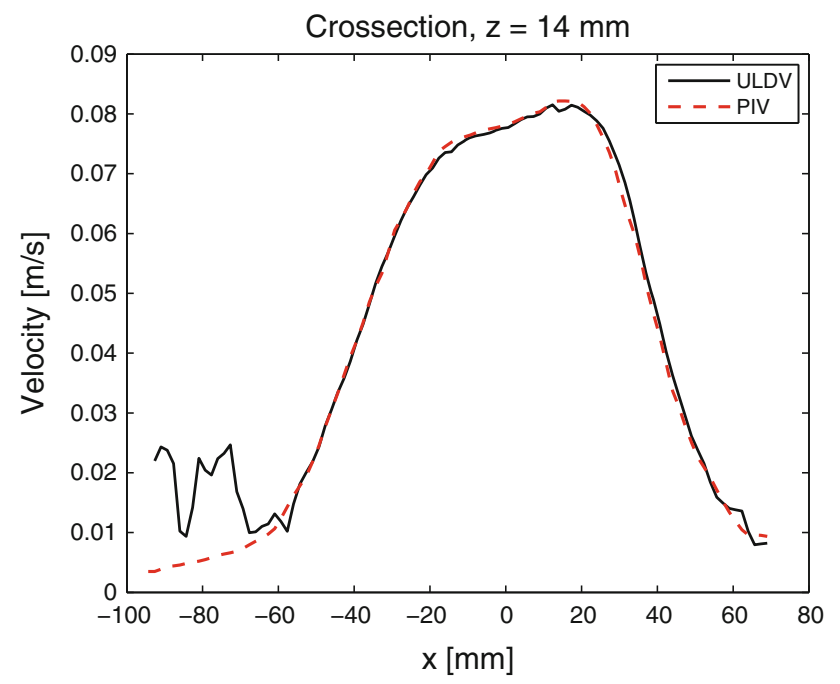

Fig. 9 Comparison of the ILDV and the PIV Measurements

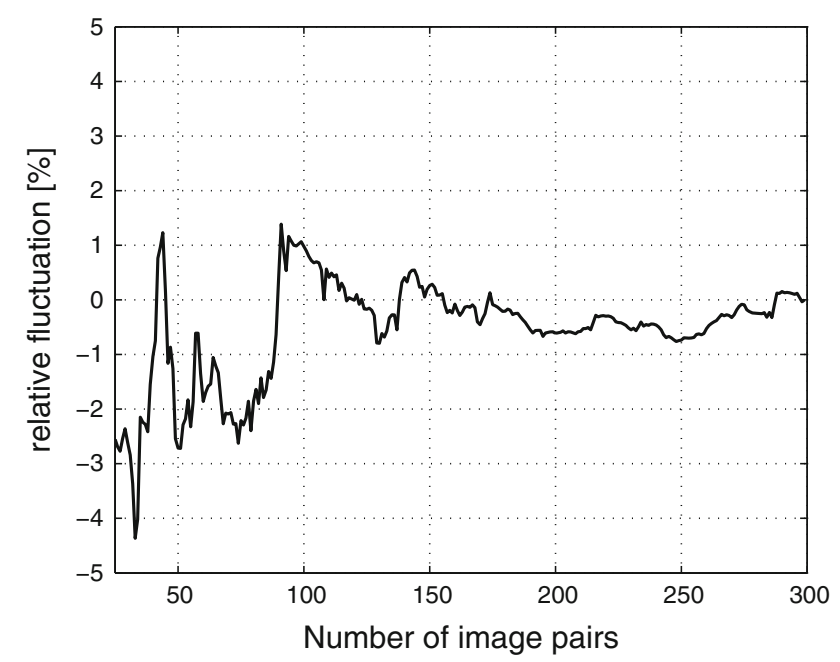

Fig. 10 Convergence of the measured velocity to its mean

The presented signal processing requires the calculation of the correlation coefficient using a number of image pairs. It is therefore of if interest how many image pairs are required for an accurate result. Figure 10 shows the convergence of the measured velocity at one point on the jet ( $x=-30 \mathrm{~mm}, z=0 \mathrm{~mm}$ ) to its mean normalized by the measurement range. With more than 150 image pairs, the fluctuations are lower than $1 \%$. Therefore, the 300 recorded image pairs are sufficient.

To perform an ILDV measurement using the high-speed camera, it had be placed closer to the SPDA to observe the flow under a shallower angle and the lens was replaced by a Nikon $50 \mathrm{~mm} f / 1.4$ lens. This was necessary since the frame rate of the high-speed camera had to be increased to $16,000 \mathrm{fps}$, which reduced the integration time by a factor of 32 compared to the previous PIV measurements. At $16,000 \mathrm{fps}$, the resolution of the high-speed camera dropped down to $512 \times 64$ pixels, but in exchange, it allowed to record 4,096 images. This way data of the HSC could be used to do PIV and ILDV simultaneously since enough images for a reasonable particle shift for the PIV analysis could be recorded. However, due to the low resolution in one direction, the PIV analysis was only possible on three lines. A comparison of the measured velocities is shown in Fig. 11. The velocities calculated using the data of the HSC show a very good agreement for the two frequency analysis algorithms (pulse-pair-based and FFT-based) for the ILDV measurement. The agreement with the PIV reference measurement is also very good, but the PIV measurement shows significant errors in the areas with zero flow velocities as well. This is due to the Doppler signal, which is superimposed onto the particle motion creating periodic intensity fluctuations in the signal. Those fluctuations can be averaged out for the PIV measurement for the high 


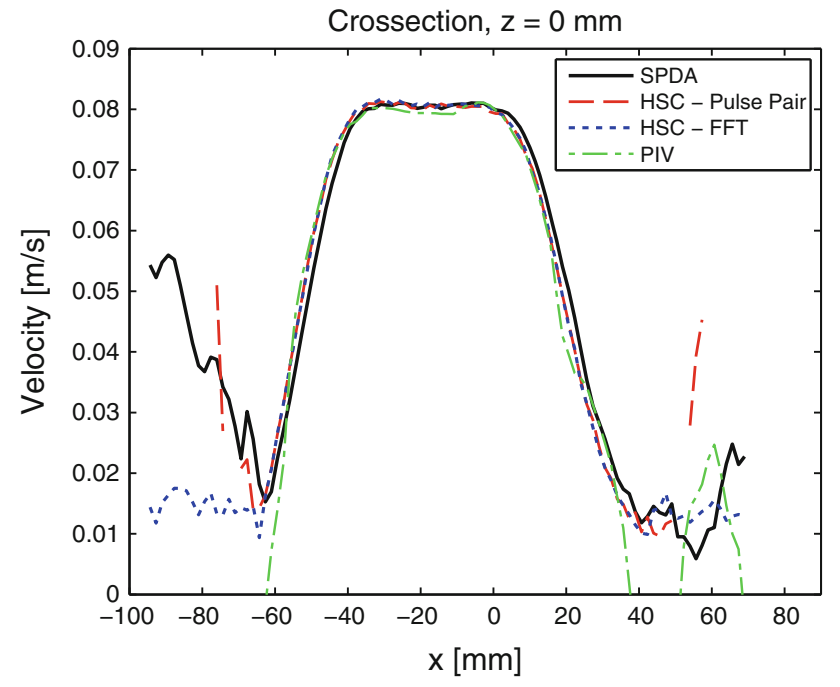

Fig. 11 Comparison of ILDV and PIV using the SPDA and the HSC

velocities by taking the mean of 30 consecutive images to form one single image for the PIV analysis. This does not work for the low velocities since the Doppler frequency is too low and those low frequency brightness fluctuations disturb the PIV analysis for the very low velocities. The velocity distribution of the SPDA is slightly shifted with respect to the data from the HSC. This shift results from a slight misalignment of the checkerboard target with respect to the light sheet during the calibration process. Apart from this shift, the agreement between the measurements using the HSC and the ILDV measurement using the SPDA is very good again.

\subsection{Dual-sensor camera system}

An ILDV system using only a single SPDA has one main problem: The algorithm to determine the frequency of the signal requires image pairs with a small time delay $\tau$ between them, limiting the velocity measurement range. This time delay between two images in a single detector system is determined by the frame rate of the camera. A system using two sensors in one camera system eliminates the problem as the two sensors can acquire the images with much smaller time delays between the two images. With smaller time delays, the crossing angle between the laser sheets can also be reduced since the time the signal needs to be persistent is reduced too. First measurements of the velocity distribution on a rotating disk using such a camera system were performed. At the same time, the issue of the noisy signal at low velocities can be avoided by premodulating the frequency of one of the light sheets as it is done in classical point measurement LDV systems to eliminate the directional ambiguity. Figure 12 shows a close-up of the camera system. The light from the scene is imaged using a Nikon $85 \mathrm{~mm} f / 1.4$ lens. Inside the camera system, a non-polarizing beam splitter (BS) divides the light for the two SPDAs. The detectors are aligned using micropositioning stages with subpixel precision relative to each other in order to assure that the corresponding pixels acquire the same signal from the scene.

Figure 13 shows the setup used for the measurement on the rotating disk. The beam of the $\mathrm{CW}$ laser is first passed into a telescope (T) to reduce its diameter and then split up into two parts using a 50:50 beam splitter (BS). One of the two resulting beams is fed into an electrooptic modulator (EOM, Conoptics 350-50) to shift the frequency of the laser beam. The two beams are then redirected using several mirrors (M), expanded and crossed using a convex lens to illuminate the rotating disk. Thus, the disk is not illuminated by a light sheet, but each point on the disk is

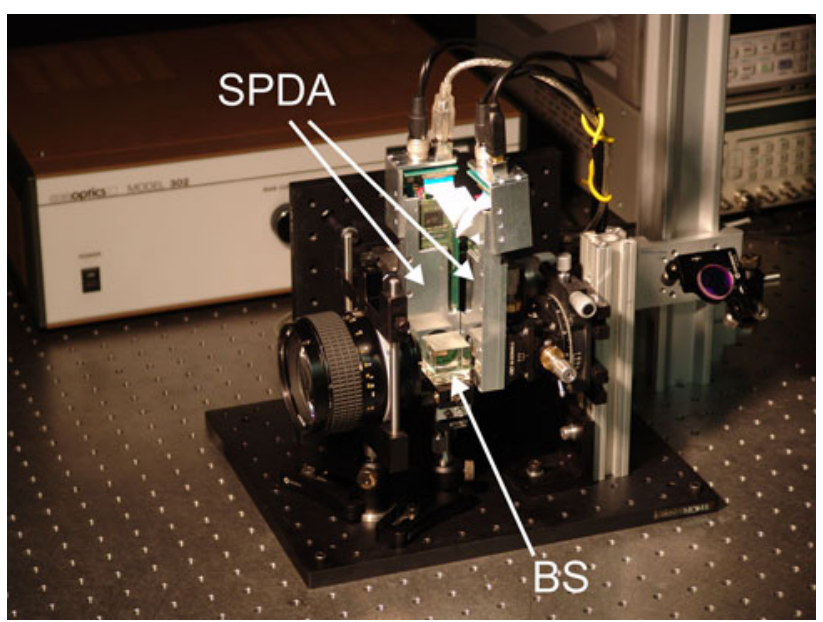

Fig. 12 Dual SPDA camera system

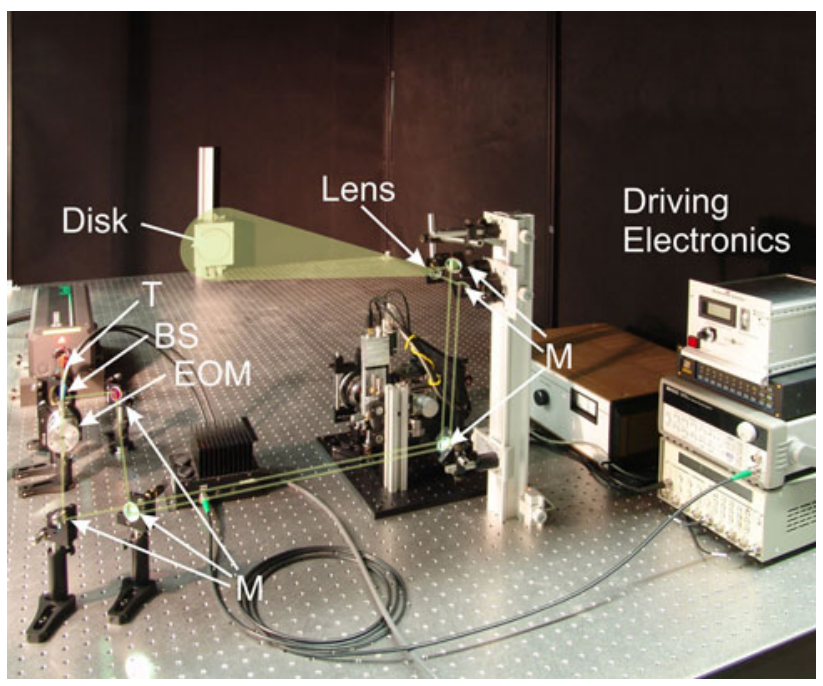

Fig. 13 Setup of the rotating disk experiment 
illuminated from two directions with a crossing angle of approx. $0.15^{\circ}$.

A close-up on the rotating disk is shown in Fig. 14, indicating the two illumination directions and the measurement area. Both illumination directions are in the $y-z$ plane. This allows to measure the $\mathrm{z}$-component of the velocity. The measured area on the rotating disk was approx. $80 \mathrm{~mm} \times 80 \mathrm{~mm}$, and the rotational frequency was set to $6 \mathrm{~Hz}$. The camera was set to a demodulation frequency of $16 \mathrm{kHz}$ and recorded 512 image pairs with a time delay of $4 T_{q p}=62.5 \mu \mathrm{s}$. The EOM was set to shift the frequency of one light sheet by $16 \mathrm{kHz}$. Therefore, signals of particles that are not moving will have an apparent Doppler frequency of $16 \mathrm{kHz}$. The demodulation frequency of the camera system and therefore the measurement range was limited by the available laser power of $5.5 \mathrm{~W}$. Higher demodulation frequencies lead to shorter integration times, and for integration times shorter than $15.625 \mu \mathrm{s}$, there was not enough light left to be detected.

Figure 15 presents the results of the measurement. It shows the expected linear distribution: The z-component of the velocity is constant on straight lines in the $\mathrm{z}$ direction and increases linearly in the $x$ direction. Due to the use of the EOM, negative velocities are measureable as well, and the noise problem at low velocities has disappeared. Figure 16 compares the true velocity of the rotating disk along a line with the measured velocity. The upper plot shows the measured velocities and the true velocity of the disk, while the lower graph shows the error of the measurement. Although all calibration parameters of the system were only measured in situ and not calibrated by an independent procedure, the measurement shows a good agreement. The calculated error is below $0.1 \mathrm{~m} / \mathrm{s}$ everywhere the disk surface, which is $<3.5 \%$ of the full measurement range of 3 $\mathrm{m} / \mathrm{s}$.

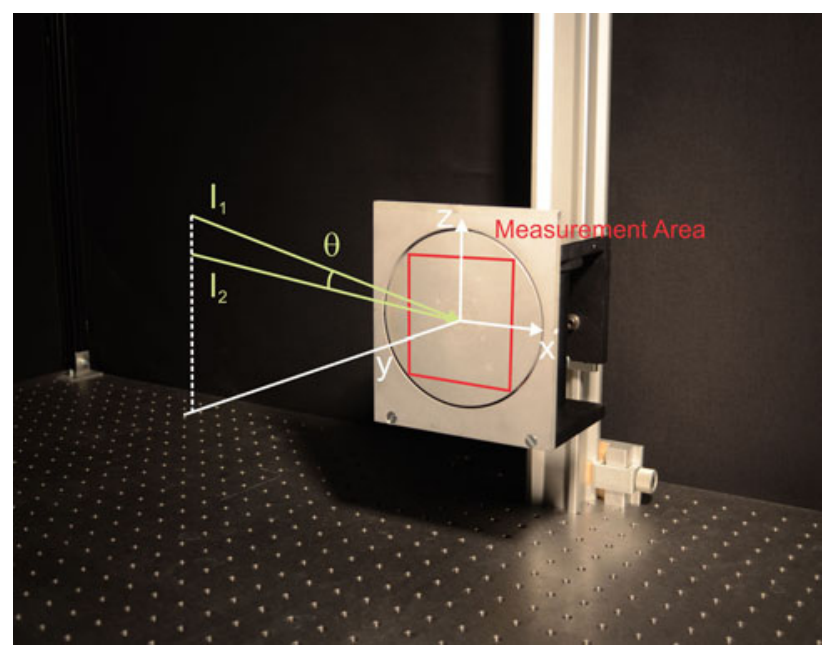

Fig. 14 Close-up on the rotating disk

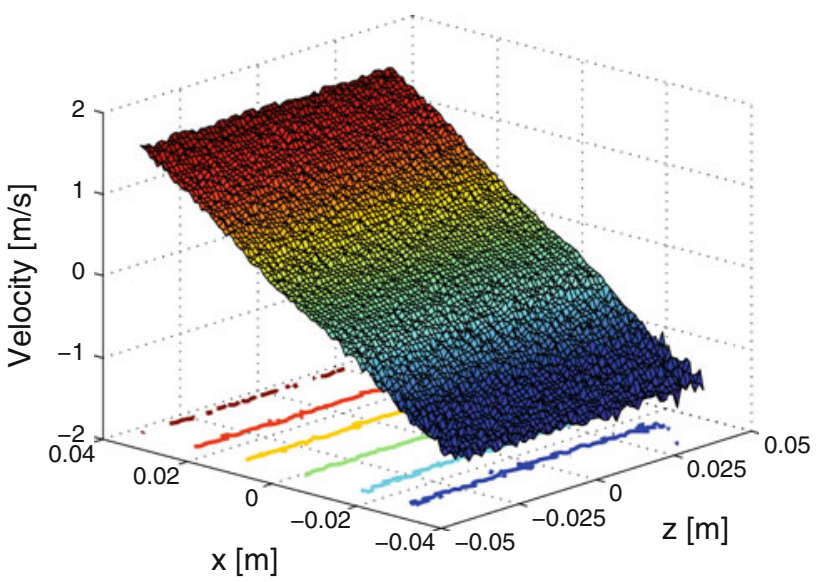

Fig. 15 Measured $z$ component of the velocity of the rotating disk

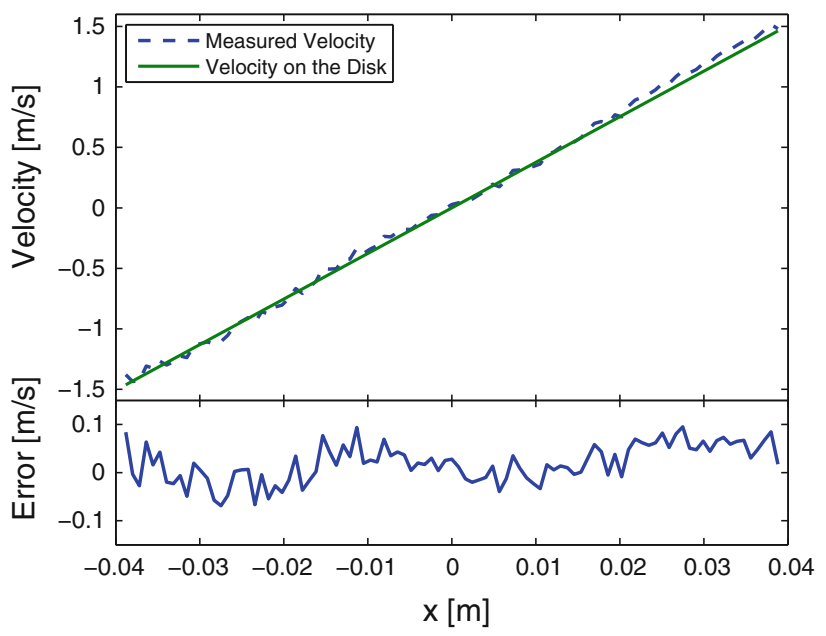

Fig. 16 Top: Comparison of the measured and the true velocity of the rotating disk. Bottom: Error of the velocity measurement

\section{Conclusions}

The jet experiment demonstrates the basic feasibility of demodulating the Doppler shift of scattered light using crossed light sheets and detecting the signal with either an imaging lock-in detector array or a high-speed camera to measure flow velocity distributions. The ILDV measurements show a good agreement with reference PIV measurements for both camera systems. Using the crossed light sheet setup, the range of measurable velocities could be extended by more than an order of magnitude compared to HDGV from $2 \mathrm{~mm} / \mathrm{s}$ to $8 \mathrm{~cm} / \mathrm{s}$. This is already sufficient for a number of applications especially when considering secondary flows.

The experiment using the two sensor camera setup and the EOM demonstrate the further potential of the technique: The measurement range could be extended to $3 \mathrm{~m} / \mathrm{s}$, and the EOM allowed to shift the operating point of the 
measurement system. Still, this velocity range can be extended further: The SPDA has only been used at a demodulation frequency of $16 \mathrm{kHz}$ and not yet at its limit of $250 \mathrm{kHz}$ due to the lack of a more powerful laser. With the SPDA running at its maximum speed, the measurement range would be extended to about $40 \mathrm{~m} / \mathrm{s}$. However, this also reduces the integration time per frame to $1 \mu \mathrm{s}$, which would require a proportional increase in laser power to $86 \mathrm{~W}$.

The ability to use a standard high-speed camera instead of the SPDA as it is shown in the jet experiment is an interesting alternative. Although high-speed cameras are more expensive and their resolution at $1 \mathrm{Mfps}$ is lower compared to the SPDA, they offer two major advantages: Using the SPDA to measure a flow field limits, the measurement technique to measuring mean flow fields since about 150-300 image pairs need to be averaged for the autocorrelation to achieve a converged result. Due to the frame rate of 6,000 fps, this will always take $25-50 \mathrm{~ms}$. Even for low-speed turbulent flows, this will be too slow to observe the turbulent structures. If instead a high-speed camera is used, the measurement of unsteady flows is also possible despite the need to record a time series of images: During the time, it takes to record the 150-300 images required for the data analysis a particle will not travel more than 5 pixels. Otherwise, the particle would not generate enough modulation cycles on a pixel for a proper frequency analysis. Thus, the signal acquisition time is in the same order of magnitude as for a PIV measurement where a particle shift of 10 pixels between two images is needed. The other advantage of high-speed cameras is their fill factor of about $50 \%$ compared to the low fill factor of only $10 \%$ of the SPDA. This reduces the power requirement for the illumination by a factor of 10 compared to the dual SPDA camera system. However, the benefits of using standard high-speed cameras for ILDV is still subject of ongoing research especially regarding turbulent flows and the out-of-plane component of the velocity.

\section{References}

Ainsworth RW, Thorpe SJ, Manners RJ (1997) A new approach to flow-field measurement: a view of Doppler global velocimetry techniques. Int J Heat Fluid Flow 18:116-130

Beer S, Seitz P (2005) Real-time tomographic imaging without X-rays: a smart pixel array with massively parallel signal processing for real-time optical coherence tomography performing close to the physical limits. Research in microelectronics and electronics $2005 \mathrm{PhD}$, vol 2, pp 135-138

Beutner TJ, Elliott GS, Mosedale A, Carter C (1998) Doppler global velocimetry applications in large scale facilities. In: AIAA 20th advanced measurement and ground testing conference, AIAA paper 98-2608

Elliott GS, Beutner TJ (1999) Molecular filter based planar Doppler velocimetry. Prog Aerosp Sci 35:799-845

Ghiglia DC, Romero LA (1994) Robust two-dimensional weighted and unweighted phase unwrapping that uses fast transforms and iterative methods. J Opt Soc Am A 11:107-117

Landolt A, Roesgen T (2009) Global Doppler frequency shift detection with near-resonant interferometry. Exp Fluids 47(4-5):733-743

Meier AH, Roesgen T (2009) Heterodyne doppler global velocimetry. Exp Fluids 47(4-5):665-672

Miller KS, Rochwarger MM (1972) A covariance approach to spectral moment estimation. IEEE Trans Info Theory 18: $588-596$

Roehle I, Schodl R, Voigt P, Willert C (2000) Recent developments and applications of quantitative laser light sheet measuring techniques in turbomachinery components. Meas Sci Technol 11:1023-1035

Samimy M, Wernet MP (2000) Review of planar multiple-component velocimetry in high-speed flows. AIAA J 38:553-574

Smith MW, Drummond JP, Northam GB (1996) Application of absorption filter planar Doppler velocimetry to sonic and supersonic jets. AIAA J 34:434-441

vom Stein HD, Pfeiffer HJ (1969) A Doppler difference method for velocity measurements. Metrologia 5:59-61

Yeh Y, Cummins HZ (1964) Localized fluid flow measurements with an $\mathrm{He}-\mathrm{Ne}$ laser spectrometer. Appl Phys Lett 4:176 